\title{
Current status of immune checkpoint inhibitors in treatment of non-small cell lung cancer
}

\author{
Sung Won Lim and Myung-Ju Ahn
}

Division of Hematology-Oncology, Department of Medicine, Samsung Medical Center, Sungkyunkwan University School of Medicine, Seoul, Korea

\author{
Received: May 19, 2018 \\ Accepted: July 20, 2018

\section{Correspondence to} \\ Myung-Ju Ahn, M.D. \\ Division of Hematology-Oncol- \\ ogy, Department of Medicine, \\ Samsung Medical Center, Sam- \\ sung Biomedical Research Insti- \\ tute, Sungkyunkwan University \\ School of Medicine, 81 Irwon-ro, \\ Gangnam-gu, Seoul o6351, Korea \\ Tel: $+82-2-3410-3438$ \\ Fax: +82-2-3410-1754 \\ E-mail: silk.ahn@samsung.com
}

Lung cancer remains a leading cause of cancer mortality worldwide, including in Korea. Systemic therapy including platinum-based chemotherapy and targeted therapy should be provided to patients with stage IV non-small cell lung cancer (NSCLC). Applications of targeted therapy, such as an epidermal growth factor receptor (EGFR) tyrosine kinase inhibitors (TKIs) and anaplastic lymphoma kinase (ALK) inhibitors, in patients with NSCLC and an EGFR mutation or ALK gene rearrangement has enabled dramatic improvements in efficacy and tolerability. Despite advances in research and a better understanding of the molecular pathways of NSCLC, few effective therapeutic options are available for most patients with NSCLC without druggable targets, especially for patients with squamous cell NSCLC. Immune checkpoint inhibitors such as anti-cytotoxic T lymphocyte antigen-4 or anti-programmed death-1 (PD-1) or programmed death-ligand 1 (PD-L1) have demonstrated durable response rates across a broad range of solid tumors, including NSCLC, which has revolutionized the treatment of solid tumors. Here, we review the current status and future approaches of immune checkpoint inhibitors that are being investigated for NSCLC with a focus on pembrolizumab, nivolumab, atezolizumab, durvalumab, and ipilimumab.

Keywords: Immune checkpoint inhibitor; Carcinoma, non-small-cell lung; Immunotherapy

\section{INTRODUCTION}

Lung cancer remains a leading cause of cancer mortality worldwide, including in Korea [1]. Non-small cell lung cancer (NSCLC) accounts for $85 \%$ of lung cancers, and its 5 -year survival rate (18\%) is much lower than that of other cancers, with survival of only $4 \%$ for advanced or metastatic disease [2]. Histology, molecular pathology, age, performance status (PS), comorbidities, and patient preferences should be considered before making decisions regarding a treatment strategy.

Systemic therapy should be provided to patients with stage IV NSCLC with PS o-2 because chemotherapy prolongs survival, improves quality of life, and improves disease-related symptoms compared to best supportive care [3]. Platinum-containing doublet chemotherapy, such as that combined with paclitaxel, gemcitabine, irinotecan, vinorelbine, or docetaxel, is considered standard therapy [4]. In contrast, pemetrexed is preferred to gemcitabine in patients with non-squamous NSCLC based on its improved survival and fewer side effects [5].

Applications of targeted therapy, such as epidermal growth factor receptor (EGFR) tyrosine kinase inhibitors (TKIs) and anaplastic lymphoma kinase (ALK) inhibitors, in patients with NSCLC and an EGFR mutation or ALK gene rearrangement has enabled dramatic improvements in efficacy and tolerability [6]. A number 
of randomized phase III studies comparing EGFR TKI or ALK TKI with platinum doublet chemotherapy in patients with advanced NSCLC have shown consistently high response rates and longer progression free survival (PFS) along with improved quality of life. These results have given rise to EGFK TKI and ALK TKI becoming the standard of care as first-line therapies in patients with NSCLC and an EGFR mutation or ALK gene rearrangement [7-12]. However, most patients develop acquired resistance after 9 to 11 months of PFS with EGFR TKI or ALK TKI, even though second- or third-line TKIs were used [13].

Despite advances in research and a better understanding of the molecular pathways in NSCLC, there are limited effective therapeutic options available for the majority of patients with NSCLC without druggable targets, particularly for patients with squamous cell NSCLC. Immune checkpoint inhibitors (CPIs), such as anti-cytotoxic T lymphocyte antigen-4 (CTLA-4), anti-programmed death-1 (PD-1), or programmed death-ligand 1 (PD-L1), demonstrate durable response rates across a broad range of solid tumors, including NSCLC, which has revolutionized treatment of solid tumors [14]. Here, we review the current status and future approaches of immune checkpoint blockades with a focus on pembrolizumab, nivolumab, atezolizumab, durvalumab, and ipilimumab, which are being investigated for treating NSCLC.

\section{MECHANISM OF IMMUNE CHECKPOINT INHIBITORS}

Immune checkpoints are orchestrated by a set of co-stimulatory and co-inhibitory molecules that regulate the activation and effector functions of $\mathrm{T}$ lymphocytes. These regulatory circuits enable self-tolerance under normal physiological conditions but frequently become co-opted during malignancy [15]. The most therapeutically relevant mechanism for immune resistance in NSCLC is considered the expression of immune inhibitory molecules in the tumor microenvironment. Among these inhibitory ligands (so called checkpoint ligands), PD-Li has been the most studied in NSCLC, although PD-L2, $\mathrm{B}_{7}-\mathrm{H}_{3}$, and $\mathrm{B}_{7}-\mathrm{H}_{4}$ have also been reported to be upregulated in lung cancer.
Increased numbers of $\mathrm{CD}_{4}+$ and $\mathrm{CD} 8+$ tumor-infiltrating T-lymphocytes are associated with a good prognosis in patients with lung cancer [16]. Immune checkpoints, represented by the interaction between the cell surface proteins CTLA-4 and PD-1 and their respective ligands, produce a negative signal for T-cells. Thus, immune checkpoint signaling decreases T-cell function, including proliferation, cytokine release, and secretion of cytotoxic granules. CTLA-4 binds to its receptors, $\mathrm{B} 7-1$ (CD80) or $\mathrm{B} 7-2$ (CD86), to give rise to a negative signal between T-cells and antigen presenting cells (APCs) in central lymph nodes. In contrast, PD-1 binds to its ligands PD-L1 (B7-H1) and PD-L2 (B7-DC) on APCs, tumor cells (TCs), and host stromal cells. Tumors grow and progress because tumors provide these immunoregulatory pathways to circumvent immune surveillance. Immune surveillance and T-cell function can be restored by blocking immune checkpoints with monoclonal antibody antagonists. Ipilimumab, a humanized immunoglobulin $\mathrm{G}$ ( $\mathrm{IgGi}$ ) monoclonal antibody that blocks CTLA-4 signaling, has been approved for treatment of malignant melanoma. Pembrolizumab and nivolumab, which are humanized IgG4 monoclonal anti-PD-1 antibodies, and atezolizumab, which is a humanized $\mathrm{IgG}_{4}$ monoclonal anti-PD-L1 antibody, have emerged as standard treatment for NSCLC. In addition, durvalumab and avelumab are anti-PD-L1 agents that are being actively investigated in clinical trials.

\section{CHECKPOINT INHIBITORS AS A SALVAGE TREATMENT IN PATIENTS WITH NSCLC PREVIOUSLY TREATED WITH PLATINUM- CONTAINING DOUBLET CHEMOTHERAPY}

Several phase 1 studies have revealed that single agent immune checkpoint blockade, such as anti-PD-1 or anti-PD-L1, shows efficacy and tolerability in refractory solid tumors, including NSCLC $[17,18]$. Therefore, multiple randomized controlled phase 3 studies were conducted to clarify the efficacy of CPIs compared with docetaxel as a salvage treatment in patients with NSCLC previously treated with platinum-based chemotherapy.

KEYNOTE-o10, a randomized controlled phase $2 / 3$ trial involving 202 academic medical centers in 24 countries, showed significantly prolonged overall sur- 
vival (OS) (14.9 months vs. 8.2 months; hazard ratio [HR], $0.54 ; 95 \%$ confidence interval [CI], 0.38 to $0.77 ; p<0.0001$ ) and PFS (5.0 months vs. 4.1 months; HR, 0.59; 95\% CI, 0.44 to $0.78 ; p=0.0001$ ) in patients treated with the anti-PD-1 inhibitor pembrolizumab $2 \mathrm{mg} / \mathrm{kg}$ compared with docetaxel among previously treated patients with at least $50 \%$ of TCs expressing PD-L1 [19].

OAK, a randomized, open-label phase 3 trial involving 194 academic or community oncology centers in 31 countries, reported results of PD-L1-targeted therapy (atezolizumab) resulting in a clinically relevant improvement in OS versus docetaxel (median 12.6 months vs. 8.9 months; HR, 0.75; 95\% CI, 0.59 to 0.96 ), regardless of PD-L1 expression or histology with a favorable safety profile [20].

In CheckMate 017 [21] and CheckMate 057 [22], which were international, randomized, open-label, phase 3 studies, patients were randomly assigned 1:1 to receive nivolumab (3 mg/kg every 2 weeks) or docetaxel (75 mg/ $\mathrm{m}^{2}$ every 3 weeks). These studies demonstrated that nivolumab provided long-term clinical benefits regard- less of PD-L1 expression [23].

In conclusion, immunotherapy may be an optimal option for salvage treatment in patients with advanced NSCLC previously treated with platinum-containing doublet chemotherapy, especially in those with high PD-L1 expression, but also in those with no or low PDL1 expression (Table 1). However, one treatment is not favorable over another in this setting because of the absence of a head-to-head comparison.

\section{CHECKPOINT INHIBITORS AS FIRST-LINE MONOTHERAPY FOR ADVANCED NSCLC}

First-line platinum-based chemotherapy remains the standard of care for patients with advanced NSCLC without an EGFR mutation or ALK rearrangement. However, the efficacy of chemotherapy remains poor, and new strategies are needed. CPIs show tremendous and durable responses as a salvage treatment in some previously treated patients with advanced NSCLC when

Table 1. Immune checkpoint blockades versus chemotherapy (docetaxel $75 \mathrm{mg} / \mathrm{m}^{2}$ every 3 weeks) as salvage treatment

\begin{tabular}{|c|c|c|c|c|c|c|}
\hline Study & Drug & Histology & $\begin{array}{c}\text { PD-L1 } \\
\text { assessment }\end{array}$ & $\begin{array}{l}\mathrm{PD}-\mathrm{L} 1 \\
\text { status }\end{array}$ & $\begin{array}{l}\text { Median OS, } \\
\text { mon (HR) }\end{array}$ & $\begin{array}{l}\text { Median PFS, } \\
\text { mon (HR) }\end{array}$ \\
\hline \multirow[t]{2}{*}{$\begin{array}{l}\text { KEYNOTE-o1O } \\
\text { (phase } 2 / 3 \text { ) }\end{array}$} & $\begin{array}{l}\text { Pembrolizumab } \\
2 \mathrm{mg} / \mathrm{kg} \text { every } 3 \text { weeks }\end{array}$ & NSCLC & $22 \mathrm{C}_{3}$ (Dako) & $\geq 1 \%$ & 10.4 vs. $8.5(0.71)$ & 3.9 vs. $4.0(0.88)$ \\
\hline & & & & $\geq 50 \%$ & 14.9 vs. $8.2(0.54)$ & 5.0 vs. $4.1(0.59)$ \\
\hline \multirow[t]{5}{*}{ OAK (phase 3) } & $\begin{array}{l}\text { Atezolizumab } \\
\text { 1,200 mg every } 3 \text { weeks }\end{array}$ & NSCLC & $\mathrm{SP}_{142}$ (Ventana) & All & 13.8 vs. $9.6(0.73)$ & 2.8 vs. $4.0(0.95)$ \\
\hline & & & & $\geq 1 \%$ & 15.7 vs. $10.3(0.74)$ & 2.8 vs. 4.1 (0.91) \\
\hline & & & & $\geq 5 \%$ & 16.3 vs. $10.8(0.67)$ & 4.1 vs. $3.6(0.76)$ \\
\hline & & & & $\geq 50 \%$ & 20.5 vs. $8.9(0.41)$ & 4.2 vs. $3.3(0.63)$ \\
\hline & & & & $<1 \%$ & 12.6 vs. $8.9(0.75)$ & 2.6 vs. $4.0(1.00)$ \\
\hline $\begin{array}{l}\text { CheckMate o17 } \\
\text { (phase 3) }\end{array}$ & $\begin{array}{l}\text { Nivolumab } 3 \mathrm{mg} / \mathrm{kg} \\
\text { every } 2 \text { weeks }\end{array}$ & $\begin{array}{l}\text { Squamous } \\
\text { NSCLC }\end{array}$ & 28-8 (Dako) & All & 9.2 vs. $6.0(0.59)$ & 3.5 vs. 2.8 (o.62) \\
\hline $\begin{array}{l}\text { CheckMate } 057 \\
\text { (phase 3) }\end{array}$ & $\begin{array}{l}\text { Nivolumab } 3 \mathrm{mg} / \mathrm{kg} \\
\text { every } 2 \text { weeks }\end{array}$ & $\begin{array}{l}\text { Non-squamous } \\
\text { NSCLC }\end{array}$ & 28-8 (Dako) & All & 12.2 vs. $9.5(0.73)$ & 2.3 vs. $4.2(0.92)$ \\
\hline \multirow[t]{4}{*}{$\begin{array}{l}\text { CheckMate } \\
\text { 017/057 }\end{array}$} & $\begin{array}{l}\text { Nivolumab } 3 \mathrm{mg} / \mathrm{kg} \\
\text { every } 2 \text { weeks }\end{array}$ & NSCLC & 28-8 (Dako) & All & 11.1 vs. 8.1 (0.72) & \\
\hline & & & & $\geq 1 \%$ & 13.4 vs. $8.5(0.67)$ & \\
\hline & & & & $\geq 5 \%$ & 17.2 vs. $7.7(0.51)$ & \\
\hline & & & & $\geq 50 \%$ & 20.6 vs. $8.0(0.42)$ & \\
\hline
\end{tabular}

PD-L1, programmed death-ligand 1; OS, overall survival; HR, hazard ratio; PFS, progression free survival; NSCLC, non-small cell lung cancer. 
compared to the second-line docetaxel. Therefore, it was assumed CPIs would produce good responses as a first-line treatment in patients with previously untreated advanced NSCLC. The results of several large randomized phase 3 trials are summarized as follows.

CheckMate 026, a randomized controlled phase 3 trial of first-line nivolumab ( $3 \mathrm{mg} / \mathrm{kg}$ every 2 weeks) compared with platinum-doublet chemotherapy (every 3 weeks for up to six cycles) in PD-L1-positive patients (PD-L1 $\geq 1 \%$ ), demonstrated that nivolumab was not associated with significantly longer PFS (4.2 months vs. 5.9 months; HR, 1.15; $p=0.25$ ) than chemotherapy among patients with previously untreated advanced or recurrent NSCLC with a PD-L1 expression level $\geq 5 \%$. OS was similar between the groups (14.4 months vs. 13.2 months; HR, 1.02) (Table 2) [24].

In contrast, KEYNOTE-024, a randomized controlled phase 3 trial of first-line pembrolizumab $(200 \mathrm{mg}$ every 3 weeks for up to 35 cycles or until documented progressive disease) compared with four to six cycles of platinum-based chemotherapy reported that those treated with pembrolizumab showed significantly lon-

Table 2. First-line immune checkpoint inhibitors in patients with advanced or recurrent NSCLC

\begin{tabular}{|c|c|c|c|c|c|c|}
\hline Study & Drug & Histology & $\begin{array}{l}\text { PD-L1 } \\
\text { status }\end{array}$ & $\begin{array}{l}\text { Median } \\
\text { OS, mon } \\
(\mathrm{HR})\end{array}$ & $\begin{array}{l}\text { Median } \\
\text { PFS, mon } \\
\text { (HR) }\end{array}$ & $\begin{array}{l}\text { Primary } \\
\text { endpoint }\end{array}$ \\
\hline \multicolumn{7}{|c|}{ Monotherapy of Immune checkpoint inhibitor } \\
\hline $\begin{array}{l}\text { CheckMate } 026 \\
\text { (phase 3) }\end{array}$ & $\begin{array}{l}\text { Nivolumab vs. } \\
\text { platinum-based chemotherapy }\end{array}$ & NSCLC & $\geq 1 \%$ & $\begin{array}{l}14.4 \text { VS. } 13.2 \\
(1.02)\end{array}$ & $\begin{array}{c}4.2 \text { vs. } 5.9 \\
(1.15)\end{array}$ & \\
\hline $\begin{array}{l}\text { KEYNOTE-O24 } \\
\text { (phase } 3 \text { ) }\end{array}$ & $\begin{array}{l}\text { Pembrolizumab vs. } \\
\text { platinum-based chemotherapy }\end{array}$ & NSCLC & $\geq 50 \%$ & $\begin{array}{l}\text { Not } \\
\text { reached } \\
(0.60)\end{array}$ & $\begin{array}{c}10.3 \text { vs. } 6.0 \\
(0.50)\end{array}$ & \\
\hline BIRCH (phase 2) & Atezolizumab (cohort $1 ; \mathrm{n}=139$ ) & NSCLC & $\geq 5 \%$ & Ongoing & Ongoing & ORR \\
\hline $\begin{array}{l}\text { IMpower } 110 \\
\text { (phase 3) }\end{array}$ & $\begin{array}{l}\text { Atezolizumab vs. platinum-based } \\
\text { chemotherapy }\end{array}$ & NSCLC & $\geq 1 \%$ & Ongoing & Ongoing & OS \\
\hline $\begin{array}{l}\text { IMpower } 111 \\
\text { (phase } 3 \text { ) }\end{array}$ & $\begin{array}{l}\text { Atezolizumab vs. platinum-based } \\
\text { chemotherapy }\end{array}$ & $\begin{array}{l}\text { Squamous } \\
\text { NSCLC }\end{array}$ & $\geq 1 \%$ & Ongoing & Ongoing & PFS \\
\hline \multicolumn{7}{|c|}{ Immune checkpoint inhibitor plus immune checkpoint inhibitor } \\
\hline \multirow[t]{2}{*}{$\begin{array}{l}\text { CheckMate } 227 \\
\text { (phase 3) }\end{array}$} & $\begin{array}{l}\text { Nivolumab plus ipilimumab vs. } \\
\text { platinum-based chemotherapy }\end{array}$ & NSCLC & All & Ongoing & $\begin{array}{l}4.9 \text { vs. } 5 \cdot 5 \\
(0.83)\end{array}$ & \\
\hline & & & $\begin{array}{l}\text { High } \\
\text { TMB }\end{array}$ & Ongoing & $\begin{array}{l}7.2 \text { vs. } 5.5 \\
(0.58)\end{array}$ & \\
\hline $\begin{array}{r}\text { MYSTIC } \\
\text { (phase 3) }\end{array}$ & $\begin{array}{l}\text { Durvalumab } \pm \text { tremelimumab vs. } \\
\text { platinum-based chemotherapy }\end{array}$ & $\begin{array}{l}\text { Non-squamous } \\
\text { NSCLC }\end{array}$ & All & Ongoing & Ongoing & PFS, OS \\
\hline \multicolumn{7}{|c|}{ Immune checkpoint inhibitor plus chemotherapy } \\
\hline $\begin{array}{l}\text { KEYNOTE-O21 } \\
\text { (phase 2) }\end{array}$ & $\begin{array}{l}\text { Pembrolizumab plus platinum-based } \\
\text { chemotherapy vs. chemotherapy } \\
\text { alone }\end{array}$ & $\begin{array}{l}\text { Non-squamous } \\
\text { NSCLC }\end{array}$ & All & $\begin{array}{l}\text { Not } \\
\text { reached } \\
(0.90)\end{array}$ & $\begin{array}{l}13.0 \text { vs. } 8.9 \\
(0.53)\end{array}$ & \\
\hline $\begin{array}{l}\text { KEYNOTE-189 } \\
\text { (phase 3) }\end{array}$ & $\begin{array}{l}\text { Pembrolizumab plus platinum-based } \\
\text { chemotherapy vs. chemotherapy } \\
\text { alone }\end{array}$ & $\begin{array}{l}\text { Non-squamous } \\
\text { NSCLC }\end{array}$ & All & $\begin{array}{l}\text { NR vs. } 11.3 \\
(0.49)\end{array}$ & $\begin{array}{c}8.8 \text { vs. } 4.9 \\
(0.52)\end{array}$ & \\
\hline $\begin{array}{l}\text { IMpower } 150 \\
\text { (phase 3) }\end{array}$ & $\begin{array}{l}\text { Atezolizumab/carboplatin/placlitaxel/ } \\
\text { bavacizumab (armB) vs. carboplatin/ } \\
\text { paclitaxel/bevacizumab (armC) }\end{array}$ & $\begin{array}{c}\text { Non-squamous } \\
\text { NSCLC }\end{array}$ & All & $\begin{array}{l}19.2 \text { vs. } 14.4 \\
(0.77)\end{array}$ & $\begin{array}{c}8.3 \text { vs. } 6.8 \\
(0.61)\end{array}$ & \\
\hline
\end{tabular}

NSCLC, non-small cell lung cancer; PD-L1, programmed death-ligand 1; OS, overall survival; HR, hazard ratio; PFS, progression free survival; ORR, objective response rate; TMB, tumor mutation burden. 
ger PFS (10.3 months vs. 6.0 months; HR, 0.50; $p<0.001$ ) and OS (not reached in either arm; HR, o.6o; $p=0.005$ ) with fewer adverse events (AEs) than those treated with chemotherapy in patients with previously untreated advanced NSCLC with PD-L1 expression on at least $50 \%$ of TCs and no sensitizing mutation of the EGFR gene or translocation of the ALK gene [25]. This robust finding led to U.S. Food and Drug Administration (FDA) approval of pembrolizumab as a first-line treatment in patients with high PD-Li expression in at least 50\% of TCs.

The KEYNOTE-042 study (NCTo2220894) was a randomized phase 3 trial comparing single agent pembrolizumab with platinum-based chemotherapy for NSCLC with both squamous and non-squamous histology and PD-L1 expression $\geq 1 \%$. Although this study demonstrated significant improvement in OS with pembrolizumab compared to chemotherapy (16.7 months vs. 12.1 months; HR, o.81) in patients with $\geq 1 \%$ PD-L1 expression, this improvement was mostly driven by patients with $\mathrm{PD}-\mathrm{L} 1$ expression $\geq 50 \%$, because there was no difference in OS in patients with $1 \%$ to $40 \%$ of PD-Li expression. It was recently announced in a press release that this study met the primary endpoint of OS [26].

BIRCH, a phase 2 trial, demonstrated clinically significant efficacy of atezolizumab monotherapy $(1,200$ mg intravenously every 3 weeks) in patients with treatment-naive advanced NSCLC and positive PDL1 expression [27]. IMpower 110 (NCTo2409342) and IMpower 111 (NCTo2409355) are ongoing phase 3 trials comparing atezolizumab with chemotherapy in patients with PD-L1 positive ( $\geq 1 \%$ on TC or immune cell with Ventana $\mathrm{SP} 142$ assay) advanced treatment-naïve NSCLC.

\section{CHECKPOINT INHIBITOR-BASED COMBINATION THERAPIES IN TREATMENT NAÏVE ADVANCED NSCLC}

CPIs show benefits for survival as a first-line monotherapy in patients with advanced NSCLC, particularly those with high PD-L1 expression, and CPI-based combination therapies have been actively studied to obtain durable responses. There are several CPI-based combination therapies, such as dual CPI + CPI, CPI + chemotherapy, CPI + EGFR TKI, or CPI + chemotherapy + vascular endothelial growth factor (VEGF) monoclonal antibody. Herein, we focused on dual CPI + CPI and CPI + chemotherapy as a first-line treatment for patients with advanced treatment-naïve NSCLC. Two representative studies to identify the efficacy of dual CPI + CPI treatment have been conducted.

The Checkmate 227 study, a randomized phase 3 trial comparing nivolumab plus ipilimumab (Ipi-Nivo) with standard platinum-based chemotherapy in patients with NSCLC and at least 1\% PD-L1 expression, recently demonstrated significant improvement in PFS with nivolumab plus ipilimumab in patients with a high tumor mutation burden (TMB) of at least 10 mutations per megabase, as tested by the FoundationOne CDx assay [28]. The median PFS was 7.2 months (95\% CI, 5.5 to 13.2 ) versus 5.5 months (HR, 0.58; $95 \% \mathrm{CI}, 4.4$ to 5.8 ; $p<0.001)$. The objective response rate was $45.3 \%$ with nivolumab plus ipilimumab and $26.9 \%$ with chemotherapy. The incidence of grade 3 or 4 treatment-related AEs was $31.2 \%$ with nivolumab plus ipilimumab versus 36.1\% with chemotherapy. In this study, the population with at least $1 \%$ PD-Lı expression received nivolumab monotherapy. No significant difference in PFS was observed between those treated with nivolumab monotherapy and those treated with chemotherapy; median PFS was 4.2 months (95\% CI, 2.7 to 8.3) with nivolumab and 5.6 months (95\% CI, 4.5 to 7.0 ) with chemotherapy (HR, 0.95; 95\% CI, 0.61 to $1.48 ; p=0.78$ ). Although OS data are not yet complete, these results indicate that TMB is an independent predictor for selected patients who would most likely benefit from the combination of nivolumab and ipilimumab. In contrast, the MYSTIC study, which compared durvalumab plus tremelimumab with platinum-based chemotherapy, did not meet the primary endpoint of PFS. The final OS analysis is pending [29].

Preclinical data show that chemotherapy induces PDL1 expression on TCs [30]. Therefore, a CPI in combination with chemotherapy shows promising efficacy as a first-line treatment in patients with advanced NSCLC. Unlike previous dual CPI + CPI treatment, the CPI + chemotherapy combination showed remarkable results.

KEYNOTE-021, a randomized, open-label, phase 2 cohort of a multicohort study, investigated the efficacy 
of the adding pembrolizumab (200 mg every 3 weeks for 2 years) to platinum-doublet chemotherapy (carboplatin and pemetrexed for four cycles followed by pemetrexed maintenance) in patients with advanced NSCLC without targetable EGFR or ALK genetic aberrations [31]. Although at least $32 \%$ of patients in the chemotherapy arm crossed over to receive pembrolizumab monotherapy with progression, as allowed by the study protocol, the combination arm showed an improved response rate (55\% vs. $29 \% ; p=0.0016)$ and PFS (13 months vs. 8.9 months; HR, $0.53 ; p=0.0102$ ) compared to chemotherapy alone. However, grade 3 to 4 treatment-related AEs were higher in the combination arm (39\% vs. $26 \%$ ), but treatment-related AEs were similar in both arms (10\% vs. $13 \%)$. Furthermore, the proportion of patients who achieved an objective response was similar in patients with PD-L1 expression $<1 \%$ and those with a score of $\geq 1 \%$. Despite the small randomized phase II trial, this combination therapy was approved by the FDA given its robust results. The confirmatory randomized phase 3 KEYNOTE-189 (NCT02578680) has been published [32]. In this study, 616 patients with metastatic NSCLC without EGFR or ALK mutations who have not received previous treatment were randomized in a 2:1 ratio to receive pemetrexed and a platinum-based drug plus either $200 \mathrm{mg}$ of pembrolizumab or placebo every 3 weeks for four cycles. This was followed by pembrolizumab or placebo for up to 35 cycles plus pemetrexed maintenance therapy. This study met the primary endpoint of OS. The estimated OS rate at 12 months was 69.2\% (95\% CI, 64.1 to 73.8) in the pembrolizumab arm versus $49.4 \%$ (95\% CI, 42.1 to 56.2 ) in the placebo arm (HR, 0.49; $p<0.001$ ). Of note, OS improved regardless of PD-L1 expression. Median PFS was 8.8 months (95\% CI, 7.6 to 9.2 ) in the pembrolizumab arm versus 4.9 months (95\% CI, 4.7 to 5.5) in the placebo arm (HR, 0.52; $p<0.001$ ). No significant difference in AEs was observed. These results are remarkable and confirmed the results of a previous phase II study, resulting in a new standard of care first-line therapy to treat NSCLC. KEYNOTE-4O7 (NCTo2775435), a randomized double-blind phase 3 study of carboplatin-paclitaxel/Nab-paclitaxel chemotherapy with or without pembrolizumab for first-line metastatic squamous NSCLC also showed a significant improvement in OS in patients treated with the pembrolizumab combination (15.9 months vs. 11.3 months;
HR, o.64).

The IMpower 150 study is a randomized phase 3 trial comparing atezolizumab/carboplatin/paclitaxel versus atezolizumab/carboplatin/paclitaxel/bevacizumab versus carboplatin/paclitaxel/bevacizumab as first-line therapy in patients with non-squamous NSCLC [33]. An interim analysis of the two arms (atezolizumab/ carboplatin/paclitaxel/bevacizumab vs. carboplatin/ paclitaxel/bevacizumab) demonstrated a median PFS of 8.3 months (95\% CI, 7.7 to 9.8) in the atezolizumab/ carboplatin/paclitaxel/bevacizumab arm versus 6.8 months (95\% CI, 6.0 to 7.1) in the carboplatin/paclitaxel/bevacizumab arm (HR, 0.617 ; $p<0.0001)$. The preliminary OS was 19.2 months (95\% CI, 16.8 to 26.1) in the atezolizumab/carboplatin/paclitaxel/bevacizum$\mathrm{ab}$ arm versus 14.4 months (95\% CI, 12.8 to 17.1 ) in the carboplatin/paclitaxel/bevacizumab arm (HR, 0.775; $p=$ 0.0262). More interestingly, the subgroup of patients with an EGFR/ALK mutation or liver metastasis showed more benefit from the atezolizumab/carboplatin/paclitaxel/bevacizumab combination (OS [not reached vs. 17.5 months for an EGFR/ALK mutation; HR, 0.54] [13.2 months vs. 9.1 months for liver metastasis; HR, 0.54]). Other combination trials, including IMpower 132 (NCTo2657434), IMpower 130 (NCTo2367781), and IMpower 131 (NCTo2367794) are ongoing and awaiting final results [34]. The use of immune CPIs as first-line therapy in NSCLC will evolve rapidly in the coming months and years as new trial results are released.

\section{ADJUVANT/CONSOLIDATION TREATMENT}

Adjuvant chemotherapy is currently recommended for patients with resected stages II and III. However, the absolute 5-year benefit is only 5\% [35]. Therefore, promising new strategies to improve the outcome of adjuvant chemotherapy are needed. Immune checkpoint inhibitors may be a good option as adjuvant, neoadjuvant, or consolidative treatments for patients with early stage NSCLC because, hypothetically, they may work optimally in the context of minimal residual disease.

PEARLS (NCTo2504372) is a currently ongoing randomized phase 3 trial to assess the benefit of pembrolizumab for patients with completely resected stage IB-IIIA NSCLC [36]. Eligible patients received pem- 
brolizumab (200 mg every 3 weeks for a maximum of 18 doses) or placebo after complete resection, followed by standard adjuvant chemotherapy. The co-primary endpoints are disease free survival in the PD-L1 strong positive subgroup and in the overall population. ANVIL (NCTo2595944) is the newest of the Adjuvant Lung Cancer Enrichment Marker Identification and Sequencing Trial (ALCHEMIST) studies investigating adjuvant nivolumab (240 mg intravenously every 2 weeks for up to 1 year) either before or after surgical resection in patients with pathologically confirmed stage IB-IIIA NSCLC [37]. Adjuvant therapy is allowed but not required. Patients are stratified by PD-L1 status $(\geq 1 \%$ or $<1 \%)$. Co-primary endpoints are OS and disease free survival. In the TOP1201 trial, a prospective phase 2 study, the safety and feasibility of using neoadjuvant chemotherapy plus ipilimumab followed by surgery as a treatment strategy for stage II-IIIA NSCLC was proven [38]. Among 13 patients treated with preoperative chemotherapy and ipilimumab followed by surgery, the most frequent complications were prolonged air leak $(15 \%)$ and a urinary tract infection (15\%). There was no apparent increased occurrence of adverse surgical outcomes for patients who also were administered ipilimumab compared to patients receiving standard of care neoadjuvant chemotherapy alone. Although the role of adjuvant treatment has not yet been proven and it is unclear whether there are differences in efficacy according to expression of biomarkers, such as PD-L1, immune checkpoint inhibitor is expected to bring clinical benefits with few toxicities.

The results of consolidation therapy in patients with locally advanced, unresectable NSCLC are interesting. In the PACIFIC (NCTo2125461) trial [39], 709 patients with stage III NSCLC who did not have disease progression after two or more cycles of platinum-based chemoradiotherapy received consolidation therapy (473 received durvalumab and 236 received placebo). The median PFS was 16.8 months (95\% CI, 13.0 to 18.0) with durvalumab versus 5.6 months ( $95 \% \mathrm{CI}, 4.6$ to 7.8 ) with placebo (HR, 0.52; 95\% CI, 0.42 to $0.65 ; p<0.001$ ). The median time to death or distant metastasis was also significantly longer in patients treated with durvalumab than in patients treated with placebo (23.2 months vs. 14.6 months; $p<0.001$ ), and safety was similar between the groups. Immune checkpoint inhibitor are expected to improve disease free survival of patients with locally advanced stage II and III NSCLC, as shown in patients with advanced or recurrent NSCLC.

\section{CONCLUSIONS}

Several CPIs, such as nivolumab, pembrolizumab, and atezolizumab, are recommended as second- or thirdline therapy in patients with advanced NSCLC who fail platinum-based chemotherapy. Given the OS advantage and fewer side effects related to CPIs, it is reasonable to treat patients with CPIs. Four treatment options can be considered as first-line treatments for NSCLC. Pembrolizumab monotherapy in patients with NSCLC with at least $\geq 50 \% \mathrm{PD}-\mathrm{L} 1$ expression; pembrolizumab plus pemetrexed/platinum chemotherapy regardless of $\mathrm{PD}$ L1 expression for non-squamous NSCLC; atezolizumab in combination with carboplatin, paclitaxel, and bevacizumab regardless of PD-Li expression for non-squamous NSCLC; and pembrolizumab plus carboplatin/ paclitaxel or nab-paclitaxel in squamous NSCLC. However, it remains unknown whether pembrolizumab plus chemotherapy is always better than pembrolizum$\mathrm{ab}$ monotherapy in patients who express high levels of PD-L1. Therefore, we need additional biomarkers to select patients who can benefit from pembrolizumab monotherapy and avoid cytotoxic chemotherapy as well as to reduce side effects related to chemotherapy and treatment cost. Based on the IMpower 150 study, a greater benefit was observed in patients with an EGFR/ ALK mutation or liver metastasis treated with atezolizumab in combination with carboplatin, paclitaxel, and bevacizumab compared to those treated with carboplatin, paclitaxel, and bevacizumab. A confirmatory phase 3 trial is warranted given the subgroup analysis results.

The combination of ipilimumab plus nivolumab in patients with a high TMB is another option as a firstline therapy. Patients with high TMB but low PD-L1 when treated with a combination of ipilimumab plus nivolumab achieved more than $40 \%$ PFS at 12 months and the response was quite durable, which is encouraging. This combination might be tolerable considering that the discontinuation rate due to AEs in the ipilimumab plus nivolumab combination arm was 17\%. However, only PFS data are currently available; 
thus, complete OS results are awaited. Furthermore, the Kaplan-Meier PFS curves still crossed in the first 3 to 6 months even in patients with high TMB treated with ipilimumab plus nivolumab compared to chemotherapy, suggesting that an additional novel approach is needed to improve treatment outcomes of patients with early progression. Although the results are quite promising, several issues remain unresolved for practical use of TMB as a biomarker in clinical practice. The platform to analyze TMB varies and the high/low cutoff value of TMB has not been standardized. The tissue availability for the TMB test might be another issue and turn-around time should also be improved.

PD-L1 expression and TMB are considered useful biomarkers to select patients who are most likely to benefit from CPIs, but other biomarkers, such as immune gene signatures, non-invasive biomarkers, and the gut microbiome, should be further investigated, but are not within the scope of this review. Consolidation of durvalumab after concurrent chemoradiotherapy significantly improved OS in patients with locally advanced NSCLC. Treatment duration in this study was only 12 months; thus, it is still unknown whether a longer duration of treatment $>12$ months provides additional benefit. In addition, concurrent treatment of CPIs with chemoradiotherapy might be better than consolidation therapy, but may increase AEs from the concurrent approach. Ongoing clinical trials will provide evidence in the near future.

A number of immunotherapy trials are ongoing. As the options for patients increase, our understanding of how best to integrate immunotherapy into lung cancer treatment will continue to evolve in the next 5 to 10 years with new trial results. Although immune check point inhibitors have significantly improved OS and showed a durable response, several issues remain, including identifying predictive biomarkers and resistance mechanisms, determining treatment strategies to overcome resistance, and identifying combination strategies to maximize efficacy. Further collaboration between clinicians and basic scientists is needed to solve these issues.

\section{Conflict of interest}

No potential conflict of interest relevant to this article was reported.

\section{REFERENCES}

1. Edwards BK, Noone AM, Mariotto AB, et al. Annual report to the nation on the status of cancer, 1975-2010, featuring prevalence of comorbidity and impact on survival among persons with lung, colorectal, breast, or prostate cancer. Cancer 2014;120:1290-1314.

2. Siegel RL, Miller KD, Jemal A. Cancer statistics, 2018. CA Cancer J Clin 2018;68:7-30.

3. Spiro SG, Rudd RM, Souhami RL, et al. Chemotherapy versus supportive care in advanced non-small cell lung cancer: improved survival without detriment to quality of life. Thorax 2004;59:828-836.

4. Ardizzoni A, Boni L, Tiseo M, et al. Cisplatin- versus carboplatin-based chemotherapy in first-line treatment of advanced non-small-cell lung cancer: an individual patient data meta-analysis. J Natl Cancer Inst 2007;99:847857.

5. Scagliotti GV, Parikh P, von Pawel J, et al. Phase III study comparing cisplatin plus gemcitabine with cisplatin plus pemetrexed in chemotherapy-naive patients with advanced-stage non-small-cell lung cancer. J Clin Oncol 2008;26:3543-3551.

6. Lindeman NI, Cagle PT, Beasley MB, et al. Molecular testing guideline for selection of lung cancer patients for EGFR and ALK tyrosine kinase inhibitors: guideline from the College of American Pathologists, International Association for the Study of Lung Cancer, and Association for Molecular Pathology. J Mol Diagn 2013;15:415-453.

7. Mok TS, Wu YL, Thongprasert S, et al. Gefitinib or carboplatin-paclitaxel in pulmonary adenocarcinoma. N Engl J Med 2009;361:947-957.

8. Fukuoka M, Wu YL, Thongprasert S, et al. Biomarker analyses and final overall survival results from a phase III, randomized, open-label, first-line study of gefitinib versus carboplatin/paclitaxel in clinically selected patients with advanced non-small-cell lung cancer in Asia (IPASS). J Clin Oncol 2011;29:2866-2874.

9. Solomon BJ, Mok T, Kim DW, et al. First-line crizotinib versus chemotherapy in ALK-positive lung cancer. N Engl J Med 2014;371:2167-2177.

10. Soria JC, Tan DSW, Chiari R, et al. First-line ceritinib versus platinum-based chemotherapy in advanced ALK-rearranged non-small-cell lung cancer (ASCEND-4): a randomised, open-label, phase 3 study. Lancet 2017;389:917929. 
11. Zhou C, Wu YL, Chen G, et al. Final overall survival results from a randomised, phase III study of erlotinib versus chemotherapy as first-line treatment of EGFR mutation-positive advanced non-small-cell lung cancer (OPTIMAL, CTONG-o802). Ann Oncol 2015;26:1877-1883.

12. Jackman D, Pao W, Riely GJ, et al. Clinical definition of acquired resistance to epidermal growth factor receptor tyrosine kinase inhibitors in non-small-cell lung cancer. J Clin Oncol 2010;28:357-360.

13. Stewart EL, Tan SZ, Liu G, Tsao MS. Known and putative mechanisms of resistance to EGFR targeted therapies in NSCLC patients with EGFR mutations: a review. Trans1 Lung Cancer Res 2015;4:67-81.

14. Thomas A, Hassan R. Immunotherapies for non-smallcell lung cancer and mesothelioma. Lancet Oncol 2012; 13:ezo1-e310.

15. Syn NL, Teng MWL, Mok TSK, Soo RA. De-novo and acquired resistance to immune checkpoint targeting. Lancet Oncol 2017;18:e731-e741.

16. Brahmer JR, Pardoll DM. Immune checkpoint inhibitors: making immunotherapy a reality for the treatment of lung cancer. Cancer Immunol Res 2013;1:85-91.

17. Brahmer JR, Drake CG, Wollner I, et al. Phase I study of single-agent anti-programmed death-1 (MDX-1106) in refractory solid tumors: safety, clinical activity, pharmacodynamics, and immunologic correlates. J Clin Oncol 2010;28:3167-3175.

18. Patnaik A, Kang SP, Rasco D, et al. Phase I study of pembrolizumab (MK-3475; anti-PD-1 monoclonal antibody) in patients with advanced solid tumors. Clin Cancer Res 2015;21:4286-4293.

19. Herbst RS, Baas P, Kim DW, et al. Pembrolizumab versus docetaxel for previously treated, PD-L1-positive, advanced non-small-cell lung cancer (KEYNOTE-010): a randomised controlled trial. Lancet 2016;387:1540-1550.

20. Rittmeyer A, Barlesi F, Waterkamp D, et al. Atezolizumab versus docetaxel in patients with previously treated nonsmall-cell lung cancer (OAK): a phase 3, open-label, multicentre randomised controlled trial. Lancet 2017;389:255265.

21. Spigel DR, Reckamp KL, Rizvi NA, et al. A phase III study (CheckMate 017) of nivolumab (NIVO; anti-programmed death-1 [PD-1]) vs docetaxel (DOC) in previously treated advanced or metastatic squamous (SQ) cell non-small cell lung cancer (NSCLC). J Clin Oncol 2015;33(15 Suppl):80o9. 22. Paz-Ares L, Horn L, Borghaei H, et al. Phase III, random- ized trial (CheckMate 057) of nivolumab (NIVO) versus docetaxel (DOC) in advanced non-squamous cell (nonSQ) non-small cell lung cancer (NSCLC). J Clin Oncol 2015;33(18 Suppl):LBA109.

23. Horn L, Spigel DR, Vokes EE, et al. Nivolumab versus docetaxel in previously treated patients with advanced non-small-cell lung cancer: two-year outcomes from two randomized, open-label, phase III Trials (CheckMate 017 and CheckMate 057). J Clin Oncol 2017;35:3924-3933.

24. Carbone DP, Reck M, Paz-Ares L, et al. First-line nivolum$\mathrm{ab}$ in stage IV or recurrent non-small-cell lung cancer. $\mathrm{N}$ Engl J Med 2017;376:2415-2426.

25. Reck M, Rodriguez-Abreu D, Robinson AG, et al. Pembrolizumab versus chemotherapy for PD-L1-positive non-small-cell lung cancer. N Engl J Med 2016;375:18231833 .

26. Lopes G, Wu YL, Kudaba I, et al. Pembrolizumab (pembro) versus platinum-based chemotherapy (chemo) as firstline therapy for advanced/metastatic NSCLC with a PDL1 tumor proportion score (TPS) $\geq 1 \%$ : open-label, phase 3 KEYNOTE-042 study. J Clin Oncol 2018;36(18 Suppl):L$\mathrm{BA}_{4}$.

27. Besse B, Johnson M, Janne PA, et al. 16LBA Phase II, single-arm trial (BIRCH) of atezolizumab as first-line or subsequent therapy for locally advanced or metastatic PD-L1-selected non-small cell lung cancer (NSCLC). Eur J Cancer 2015;51 Suppl 3:S717-S718.

28. Hellmann MD, Ciuleanu TE, Pluzanski A, et al. Nivolumab plus ipilimumab in lung cancer with a high tumor mutational burden. N Engl J Med 2018;378:2093-2104.

29. Peters S, Antonia S, Goldberg SB, et al. 191TiP: MYSTIC: a global, phase 3 study of durvalumab (MEDI4736) plus tremelimumab combination therapy or durvalumab monotherapy versus platinum-based chemotherapy (CT) in the first-line treatment of patients (pts) with advanced stage IV NSCLC. J Thorac Oncol 2016;11(4 Suppl):S139-S140.

30. Liu WM, Fowler DW, Smith P, Dalgleish AG. Pre-treatment with chemotherapy can enhance the antigenicity and immunogenicity of tumours by promoting adaptive immune responses. Br J Cancer 2010;102:115-123.

31. Langer CJ, Gadgeel SM, Borghaei H, et al. Carboplatin and pemetrexed with or without pembrolizumab for advanced, non-squamous non-small-cell lung cancer: a randomised, phase 2 cohort of the open-label KEYNOTE-O21 study. Lancet Oncol 2016;17:1497-1508.

32. Gandhi L, Rodriguez-Abreu D, Gadgeel S, et al. Pembroli- 
zumab plus chemotherapy in metastatic non-small-cell lung cancer. N Engl J Med 2018;378:2078-2092.

33. Reck M, Socinski MA, Cappuzzo F, et al. LBA1_PR Primary PFS and safety analyses of a randomized phase III study of carboplatin + paclitaxel +/- bevacizumab, with or without atezolizumab in $1 \mathrm{~L}$ non-squamous metastatic nsclc (IMPOWER150). Ann Oncol 2017;28(Suppl 11):mdx760.002.

34. Lynch TJ, Bondarenko I, Luft A, et al. Ipilimumab in combination with paclitaxel and carboplatin as first-line treatment in stage IIIB/IV non-small-cell lung cancer: results from a randomized, double-blind, multicenter phase II study. J Clin Oncol 2012;30:2046-2054.

35. Zhong C, Liu H, Jiang L, Zhang W, Yao F. Chemotherapy plus best supportive care versus best supportive care in patients with non-small cell lung cancer: a meta-analysis of randomized controlled trials. PLoS One 2013;8:e58466.
36. Paz-Ares L, Hasan B, Dafni U, et al. 71TiP A randomized, phase 3 trial with anti-PD-1 monoclonal antibody pembrolizumab (MK-3475) versus placebo for patients with early stage NSCLC after resection and completion of standard adjuvant therapy (EORTC/ETOP 1416-PEARLS). Ann Oncol 2017;28(Suppl 2):mdxo85.013.

37. Chaft JE, Dahlberg SE, Gerber DE, et al. EA5142 adjuvant nivolumab in resected lung cancers (ANVIL): the newest study in the ALCHEMIST platform. J Clin Oncol 2017;35(15 Suppl):TPS8575.

38. Yang CJ, McSherry F, Mayne NR, et al. Surgical outcomes after neoadjuvant chemotherapy and ipilimumab for nonsmall cell lung cancer. Ann Thorac Surg 2018;105:924-929.

39. Antonia SJ, Villegas A, Daniel D, et al. Durvalumab after chemoradiotherapy in stage III non-small-cell lung cancer. N Engl J Med 2017;377:1919-1929. 